Ecole Evry Schatzman 2010: Star Formation in the Local Universe

Eds. C. Charbonnel \& T. Montmerle

EAS Publications Series, 2011

\title{
STAR FORMATION IN SPIRAL ARMS
}

\author{
Bruce G. Elmegreen ${ }^{1}$
}

\begin{abstract}
The origin and types of spiral arms are reviewed with an emphasis on the connections between these arms and star formation. Flocculent spiral arms are most likely the result of transient instabilities in the gas that promote dense cloud formation, star formation, and generate turbulence. Long irregular spiral arms are usually initiated by gravitational instabilities in the stars, with the gas contributing to and following these instabilities, and star formation in the gas. Global spiral arms triggered by global perturbations, such as a galaxy interaction, can be wavemodes with wave reflection in the inner regions. They might grow and dominate the disk for several rotations before degenerating into higher-order modes by non-linear effects. Interstellar gas flows through these global arms, and through the more transient stellar spiral arms as well, where it can reach a high density and low shear, thereby promoting self-gravitational instabilities. The result is the formation of giant spiral arm cloud complexes, in which dense molecular clouds form and turn into stars. The molecular envelops and debris from these clouds appear to survive and drift through the interarm regions for a long time, possibly $100 \mathrm{Myr}$ or more, with lingering spontaneous star formation and triggered star formation in the pieces that are still at high-pressure edges near older HII regions.
\end{abstract}

\section{Introduction}

An important feature of many disk galaxies is their spiral structure, which, for the Milky Way, has been connected with star formation since Morgan. Whitford \& Code (1953) found concentrations of OB stars in the Sagittarius spiral arm of the Milky Way. This connection suggests that spiral arms trigger star formation, which makes us wonder how such triggering might fit in with the global star formation laws discussed in the previous lecture. The answer is that spiral arms have very little influence on large-scale star formation rates, but they do organize the star

\footnotetext{
${ }^{1}$ IBM T. J. Watson Research Center, 1101 Kitchawan Road, Yorktown Heights, New York 10598 USA, bge@us.ibm.com
} 
formation in a galaxy. This is probably because most of the gas that can turn molecular, i.e., inside shielded regions at normal interstellar pressure, has already done so in the main disks of galaxies, and because this molecular gas is already forming stars as fast as it can. Star formation in this case may be viewed as saturated (Elmegreen, 2002). Additional cloud collisions in spiral shocks, or new cloud formation in spiral arms, does not add much to the molecular mass and star formation- it only moves it around. This may not be true in the outer parts of galaxies, where the gas is highly atomic. There, dynamical processes such as spiral arms could affect the average star formation rate. There is very little known about outer disks yet, so the influence of outer spiral arms on average star formation rates remains an open question.

In this lecture, we begin with a description of spiral waves and the various theories for them. Then we discuss detailed models for how spiral arms interact with the gas and affect the formation of giant clouds. We also discuss the interarm clouds and the apparent aging and destruction of dense clouds as they move to the next arm.

\section{Spiral Waves and Modes}

Bertil Lindblad (1962) noticed that $\Omega-\kappa / 2$ for angular rotation rate $\Omega$ and epicyclic frequency $\kappa$ was about constant with radius in galaxy disks. He suggested that spirals are fixed patterns with an angular rotation rate $\Omega-\kappa / 2$, moving through a disk of stars with a radial variation in the stellar rotation rate $\Omega$. Thus stars and gas move through the spiral pattern. This was the beginning of spiral density wave theory, although it was not quite right yet. Lindblad showed rotation curves for 3 galaxies, $\Omega$ versus $\kappa$, and a nearly constant $\Omega-\kappa / 2$. Rotation curves were difficult measurements at that time, and derivatives in the rotation curves, as in the evaluation of $\kappa$, were highly inaccurate.

The main problem with Lindblad's theory was that it had no forcing. Also, $\Omega-\kappa / 2$ is not quite constant. Lin \& Shu (1964) introduced a more dynamically correct spiral density wave theory. They realized that $\Omega-\kappa / 2$ could be forced by the spiral's gravity to a radial-constant value, even if it was not constant from the average rotation curve. Then the stellar orbits could be closed for a wide range of radii at a fixed pattern speed. The angular pattern speed would be slightly different from $\Omega-\kappa / 2$, and where it equaled this value, there would be a resonant interaction between the forcing from the spiral and the stellar epicyclic motions. This resonance would absorb wave energy and put it into random stellar motions, causing the wave to stop propagating at this place. This position became known as the inner Lindblad resonance. Another resonance position is where $\Omega+\kappa / 2$ equals the spiral pattern speed. This is the outer Lindblad resonance. Other resonances at $\Omega-\kappa / 3$ and $\Omega+\kappa / 3$, occur as well, limiting the range for three-arm spirals in this case. There are similar limits for 4 arm spirals, etc., and finally the last resonance where $\Omega$ itself equals the pattern speed. This is the corotation resonance, where the same stars are always inside the wave crest, following it around at the same angular speed. 
The Lin-Shu mechanism works because just inside an arm, spiral gravity pulls a star outward for short time, slowing it down a little as it rises in its epicyclic path. Just outside an arm, spiral gravity pulls the star inward, speeding it up as it falls inward in its epicyclic motion. These slow-downs and speed-ups cause the ends points of each epicycle to advance a little, closing the orbits in a rotating frame with a rate $\Omega_{p}$ such that $\Omega-\kappa / 2<\Omega_{p}<\Omega+\kappa / 2$. The gravitational effect can be seen in the dispersion relation written by Toomre (1969):

$$
(\omega-m \Omega[r])^{2}=\kappa^{2}(r)-2 \pi G \Sigma(r) k \mathcal{F}(\chi)
$$

where $\omega$ is the rate of change of the spiral phase in a fixed coordinate system, equal to $m$ times the pattern speed, $m$ is the number of symmetric spiral arms, $\Sigma$ is the mass column density in the disk, $k$ is the wavenumber, and $\mathcal{F}$ is an integral over stellar motions that depends on $\chi=k^{2} \sigma_{\mathrm{u}}^{2} / \kappa^{2}$ for rms radial speed of the stars $\sigma_{\mathrm{u}}$. Lindblad's theory did not have the last term on the right, which is from disk gravity. Traveling waves exist for Toomre parameter $Q>1$, i.e., for disks that are stable to radial perturbations.

Toomre (1969) noted that although the Lin-Shu dispersion relation for spiral waves has a phase velocity equal to the proposed pattern speed, it also has a group velocity which causes the wave crests to move inward, i.e., the spirals wrap up. Thus the "quasi-stationary" spiral density wave theory of Lin, Shu, Roberts, Yuan, and other collaborators at that time, did not work as they originally proposed. Toomre showed that for a flat rotation curve, disk-dominated gravity, and constant stability parameter $Q$, the Lin-Shu dispersion relation becomes relatively simple,

$$
(\omega-m \Omega) / \kappa=\left(m / 2^{1 / 2}\right)\left[\left(r / r_{\mathrm{CR}}\right)-1\right]
$$

for radius $r$ and corotation radius $r_{\mathrm{CR}}$. In this case, the time derivative of the dimensionless wavenumber increases at a rate equal to half the rate of change in the phase, $\omega / 2$. When the wavenumber increases with time, the spirals get closer together, which means they migrate inward. This is a fast migration, almost as fast as purely material arms would wrap up from shear.

Toomre (1969) proposed that spirals are not quasi-stationary, but transient, provoked either by interactions (Toomre \& Toomre, 1972) or noise (Toomre \& Kalnais, 1991). Kormendy \& Norman (1979) noted that "grand design" spirals are either in barred galaxies, in the rising parts of rotation curves (where $\Omega-\kappa / 2 \sim 0$ ) or in interacting galaxies. This would be consistent with Toomre's picture. Toomre (1981) identified the cause of transient spirals as "swing amplified instabilities." Many groups have studied these instabilities numerically (e.g., Fuchs et al., 2005).

The quest for a theory of quasi-stationary spiral structure was not over, though. Mark (1974), Lau et al. (1976), and Bertin et al. (1989) proposed a "modal theory" in which inward-moving waves reflect or refract off of a bulge or bar and come back out as leading (WASER2; reflection) or trailing (WASER1; refraction) spiral arms. When they reach the corotation resonance moving outward, they amplify. Part of the wave then turns around to come back in and another part of the wave keeps going outward. The result is a standing wave pattern, amplified from initial 
disk noise at corotation and forming a long-lived grand-design spiral. The corotation radius is where the outward-moving wave meets the inward-moving wave on the opposite side of the galaxy for a two-arm spiral. If the outward moving wave is leading, then at the meeting place, the swing amplifier can transform this leading wave into a strong trailing wave. For example, an inward moving wave starting at corotation in one arm of a two-arm spiral can reflect off of a bulge and move back out as a leading wave until it meets the other arm at the same radius where it started. It amplifies as it is converted into a trailing arm, adds to the original trailing arm, and then a stronger trailing arm comes in again. Trailing waves that start at different radii will not reflect and meet the opposite arm at the same radius, and so will not add to the original wave after amplification. Thus, out of all the disk noise and small spirals that they initiate, only the spiral with the ability to amplify reflected or refracted waves and reinforce itself will grow. This defines the corotation radius. Bertin et al. (1989) described this process in detail.

Spiral wave modes could exhibit an interference pattern between the inward and outward moving waves. Interference acts to modulate the amplitudes of the main arms or it may introduce slight phase shifts in the main arms. Such modulation is present in the model solutions shown by Bertin et al. (1989). Elmegreen et al. (1992) reported such interference patterns, but a more modern analysis is needed.

The various theories of spiral wave formation may be reduced to four basic types: random and localized swing-amplified spirals that are primarily in the gas (because the stellar disk is somewhat stable); random and localized swing-amplified spirals that are in the stars and the gas together; transient global waves that are in the stars and gas, and standing wavemodes that are in the stars and gas. The first type produces flocculent spiral arms and a smooth underlying stellar disk (e.g., NGC 5055), the second type produces multiple stellar and gaseous arms (e.g., NGC 3184), the third type produces long spiral arms in the stars and gas (e.g., NGC 628), and the fourth type produces strong two-arm spirals in the stars and gas, usually in response to some global perturbation like a galaxy interaction (e.g., M51, M81). Aside from M81, these galaxy examples were chosen from the THINGS survey (Walter et al., 2008).

A spiral wavemode may be compared to the pure-tone ringing of a bell after some multi-frequency impact disturbs it (e.g., it is hit by a hammer or bowed by a violin string). Random swing-amplified spirals have been called spiral chaos. They are the primary response to gravitational instabilities in the stars and gas and therefore have a strong connection with star formation and the origin of interstellar turbulence in the absence of global wavemodes (e.g., Thomasson et al., 1992; Bournaud et al., 2010). Global spiral waves or wavemodes also have a connection with star formation because of the way they force the gas into a dense molecular phase in the dust lanes (which are shock fronts) and organize it to follow the underlying stellar spiral. 


\section{Motions in Spiral Arms}

Because of the forcing from gravity, spiral arms induce a reverse shear in the stellar rotation, slowing down the stars on the inner parts and speeding up the stars on the outer parts of each arm Roberts (1969). This reverse often cancels the normal shear from average orbital motions, and makes an arm that has very little internal shear. The arm forcing from gravity also pulls everything in the arm toward the center of the arm, i.e., in a convergent manner, which is opposite to the tidal force from the surrounding galaxy. Thus spiral arms also have reduced galactic tidal forces (Elmegreen, 1992). These conditions are good for the formation of large cloud complexes, which are only weakly bound at the start. Giant clouds that form by gravitational instabilities in spiral arms do not immediately shear out into little spirals, and this allows them to grow. When they emerge from the arm, the shear rate and tidal disruption rate increase a lot, and the low-density parts of the clouds can come apart. They can also form feathers and spurs. Such feathering is commonly seen (La Vigne et al., 2006). Feathers occur primarily in grand design (2-arm) galaxies with prominent dust lanes. The feathers are closer where gas density is highest, as expected for gravitational instabilities. Their separation is $5-10$ Jeans lengths in the dust lane (La Vigne et al., 2006).

The peculiar motions from spiral arm gravity and Coriolis forces cause the gas and stars to stream along the spiral arms when they are in the arms, and to expand away from the arms when they are between the arms. Streaming motion of the gas can be very strong, perhaps $50 \mathrm{~km} \mathrm{~s}^{-1}$ or more, as in M51 (Shetty et al., 2007). Radial streaming changes sign at corotation and the observation of this allows one to locate the corotation resonance radius (e.g., Elmegreen et al., 1998). Streaming motions also allow one to measure the timing of the star formation response to the spiral arm (Tamburro et al., 2008). The streaming pattern for gas tends to be inward inside the main parts of the arms inside the corotation radius, and outward in the interarms inside corotation. This pattern of radial motions relative to the arms reverses outside of corotation. Thus the gas and star formation in outer disk spirals, as viewed, for example, by GALEX, should be streaming with positive galacto-centric radial velocities, whereas the gas and star formation in inner disk spirals should be streaming with negative galacto-centric radial velocities. Shetty et al. (2007) found a net radial inward streaming flux for the inner part of M51, including both the arms and the interarms. They suggested that this meant a non-steady spiral pattern.

\section{Magnetic Fields in Spiral Arms}

The magnetic fields of spiral galaxies have been extensively observed, particular by Rainer Beck and collaborators (e.g., Braun et al., 2010) using Faraday rotation. In NGC 6946 (Beck, 2007), the field structure is uniform in the interarm regions, probably from the combing action of shear with little disruption from star formation. It is more chaotic in the arms, and even weaker on average in the arms than the interarms because of the strong random component in the arms. The 
total field strength in the arms should be higher than in the interarms because of compression from the spirals, but this higher field strength might not be seen with rotation measures if the field direction fluctuates on small scales.

Gravitational instabilities in spiral arm gas are enhanced by the lack of shear and the magnetic field (Elmegreen, 1987), which tends to run parallel to the arms in the direction of the unstable flow. This field removes angular momentum from a growing condensation, as mentioned in Lecture 1. Kim \& Ostriker (2001) have dubbed this the Magneto-Jeans instability, and modeled it numerically. Gas collapses along spiral arms into giant cloud complexes, aided by the parallel magnetic field. The dense gas then emerges downstream from the arms as interarm feathers.

\section{Gravitational Instabilities in Spiral Arms}

Gas rich galaxies with weak stellar spirals have more interarm gas and star formation than galaxies with strong stellar spirals. In weak-arm spiral galaxies, local swing-amplified instabilities in the gas become prominent and these can occur almost anywhere. In galaxies with strong, global stellar waves, the magneto-Jeans instability forms giant cloud complexes primarily in the spiral arms, where the density is high and the shear is low.

Figure 1] shows a Hubble Space Telescope image of the galaxy NGC 4414, which has numerous patches of star formation in the midst of a faint 2-arm structure. This is an example of the first type mentioned in the previous paragraph. Figure 2 shows an HST image of M51, a strong two-arm spiral with little star formation between the stellar arms. This is an example of the second type.

Stellar spirals define two scales, $2 \pi G \Sigma / \kappa^{2}$, which is the "Toomre length"

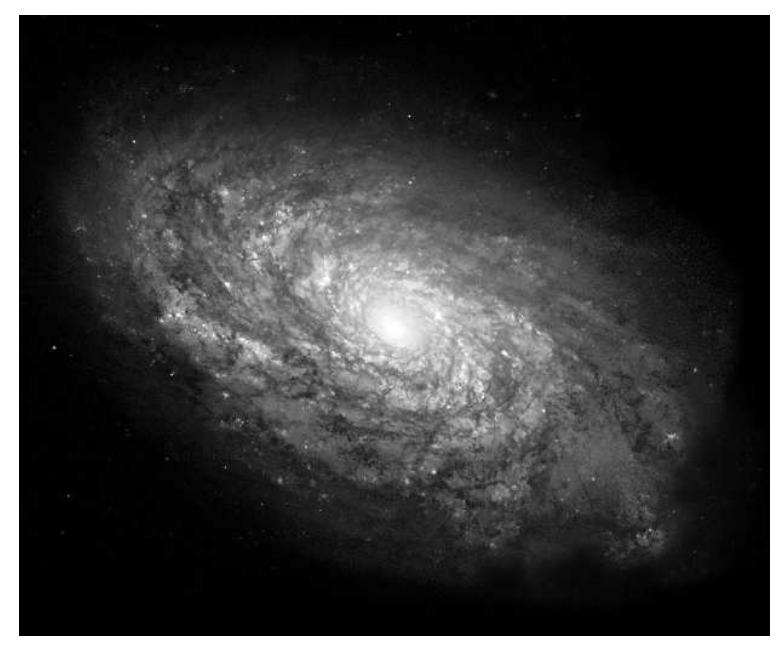

Fig. 1. Hubble Space Telescope image of NGC 4414, from multiple passbands. 
(Toomre, 1964) separating the spirals, and $2 \sigma^{2} / G \Sigma$, which is the Jeans length separating the condensations in the spirals. The Jeans length is about three times the arm width and physically smaller inside the dense dust lanes. The beads on a string seen in spiral arms are giant star complexes. Each has a feather or spur of dust from a spiral wave flow downstream. This is clearly visible in the HST image of M51, shown in Figure 2, In the interarm regions of M51, young stars are still in star complexes that are aging. Lingering star formation and triggered star formation occur in the interarm fields of cloudy debris. Further downstream, the cloud envelopes are more diffuse but there is still a little star formation in some of them (Fig. 3). The molecular envelopes of GMCs must be long-lived to survive as far as they do downstream from the arms, $\sim 100 \mathrm{Myr}$ or more. This seems to require magnetic support in the cloud envelopes (Elmegreen, 2007). Further downstream, almost at the next spiral arm, the cloudy debris from the previous arm has little associated star formation. There appears to be a lot of diffuse molecular gas indicated by these interarm dust features. They coagulate into a dust lane when they reach the next arm.

At a very basic level, a gravitational instability in a spiral arm, or in a spiral arm dust lane (Elmegreen, 1979) can be viewed as an instability in a cylinder.

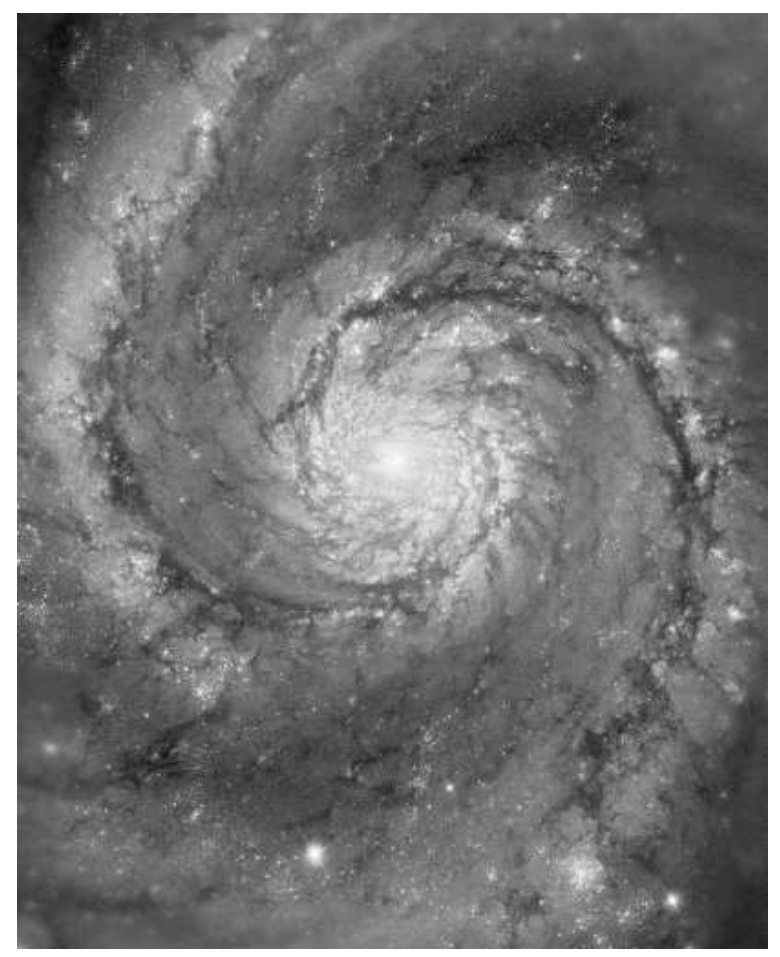

Fig. 2. Hubble Space Telescope image of NGC 5194, from multiple passbands. 
Such instabilities occur when

$$
\pi G \mu / \sigma^{2} \geq 1
$$

where $\mu$ is the mass per unit length in the cylinder and $\sigma$ is the velocity dispersion. The fastest growing mode has a wavelength of about 3 times the cylinder width (Elmegreen \& Elmegreen, 1983).

There is also enhanced star formation at the end of some strong bars. This is most likely a crowding effect from the gas that turns a corner there in its orbit relative to the bar (Lord \& Kenny, 1991). The inner, nearly straight, dust lanes in many bars do not contain much star formation and look non-self gravitating. This is probably because of high shear and radial tidal forces. Inside this dustlane, in the center of the bar, there is often a ring close to the inner Lindblad resonance (Buta \& Combes, 1996). This ring also has two characteristic scales, the thickness in the radial direction and the Jeans length. ILR rings develop major

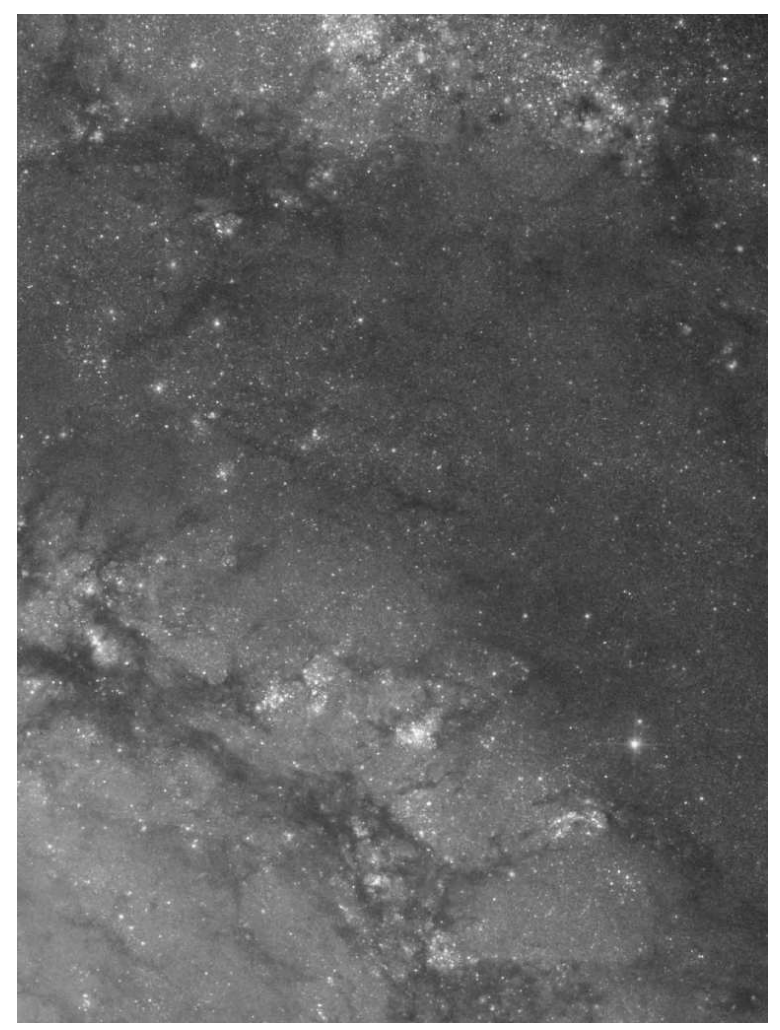

Fig. 3. Enlargement of the Western interarm region of M51, from the Hubble Space Telescope image. Dark dust clouds with small amounts of star formation, or no evident star formation, are seen. Some are at the edges of old OB associations and may contain triggered star formation. 
sites of star formation, or "hot-spots" along them, with a separation of around the Jeans length, probably because of local gravitational instabilities in the gas (e.g., Elmegreen, 1994).

\section{Star Formation in Spiral Arms}

What is the relationship between HI, CO and star formation in spiral arms? The gas is generally compressed more than the stars in a spiral density wave or swingamplified transient spiral, and if star formation follows the gas, then the blue light from star formation will be enhanced more than the yellow and red light from old stars. This makes the spirals arms blue. The Bigiel et al. (2008) and Leroy et al. (2008) correlation between SF rate and CO, which is a very tight correlation, implies that there is little difference in the rate per unit $\mathrm{CO}$ molecule for gas in strong-arm galaxies compared to gas in weak-arm galaxies.

The morphology of gas in the arms tells something about the star formation process. Grabelsky et al. (1987) showed that most of the CO clouds in the Carina arm of the Milky Way are clustered together in the cores of $10^{7} M_{\odot}$ HI clouds. Elmegreen \& Elmegreen (1987) found the same for the Sagittarius spiral arm. Lada et al. (1988) observed a similar HI envelope-CO core structure in a piece of a spiral arm in M31. Engargiola et al. (2003) showed a complete map of M33 with numerous $\mathrm{CO}$ clouds in the cores of giant $\mathrm{HI}$ clouds. The presence of giant $\mathrm{HI}$ clouds in spiral arms has been known for a long time (e.g., for the Milky Way: McGee \& Milton 1964; for NGC 6946: Boulanger \& Viallefond 1992). Now it looks like most dense molecular clouds are in the cores of giant spiral arm HI clouds, or if the gas is highly molecular at that radius in the disk, in the cores of giant clouds that are also highly molecular. This means that GMCs form by condensation inside even larger, lower-density clouds. The large HI/CO clouds, in turn, probably form by gravitational instabilities in the spiral arm gas, particularly in the dust lanes where the spiral shock brings the gas to a high density. Recall from Lecture 1 that the largest unstable clouds have the Jeans mass in a galaxy disk, given the observed turbulent speed and column density (i.e., $M \sim \sigma^{4} / G^{2} \Sigma_{\text {gas }} \sim 10^{7} M_{\odot}$ ).

In the Milky Way and M33, giant spiral arm clouds are mostly atomic, but in M51, they are mostly molecular (Ranf \& Kulkarni, 1990). This difference is presumably because the arms in M51 are much stronger than the arms in the Milky Way and M33, and the gas is denser overall in M51 as well. Thus, the pressure is higher in M51, particularly in the arms, and the gas is more highly molecular there and everywhere else in the inner disk. The physical process of giant cloud formation should be the same in all three cases, however.

Gravitational instabilities also seem to initiate cloud and star formation on the scale of whole galaxies. This process is clear in many regions, such as Stephans quintet (Mendes de Oliveira et al., 2004), NGC 4650 (Karataeva et al., 2004), and in the tidal arcs of NGC 5291 (Bournaud et al. 2007), where there are massive condensations in tidal features.

Dobbs \& Pringle (2009) studied gravitationally bound clouds in an SPH simulation. In the spiral arms, large regions formed by gravitational instabilities where 
gravity balanced thermal, turbulent and magnetic energies. When they used a star formation rate equal to $5 \%$ of the bound gas column density divided by the dynamical time, plotted versus the total gas column density, they reproduced the Kennicutt (1998) and Bigiel et al. (2008) star formation laws over the range of overlap. They noted that the star formation law is linear with column density because the dynamical time inside each bound cloud is the same, i.e., they all have the same density. They answered the long-time question of whether density waves trigger star formation (Elmegreen \& Elmegreen, 1986) by saying, no, there is no correlation between the average column density of star formation and the spiral arm potential depth. The reason is that stronger spiral waves make clouds with higher velocity dispersions and they are harder to bind into gravitating cloud complexes. The fraction of the bound gas in spiral arms increases with the spiral strength, but not the star formation rate.

Observations of spiral arm star formation and gas distributions also suggest there is little triggering (Foyle et al., 2010). The primary effect of the spiral is to concentrate the gas in the arms without significantly changing the star formation rate per unit gas.

\section{Summary}

Spirals can, in principle, be of 4 types: (1) Transient gravitational instabilities in the gas, causing "flocculent spirals," with too much stability in the stellar disk to give prominent stellar spiral waves. (2) Transient gravitational instabilities in the stars, with the gas adding force and following the stars. The gas and stars move through these transient spirals a little, but not around from arm to arm in a full circle as in idealized global stellar modes and waves. (3) Global stellar waves that are non-steady with a pattern speed that varies with radius and whose patterns wrap up toward the center over time. Stars and gas move through these spirals. (4) Global stellar wave modes that are "standing waves," with a uniform pattern speed between the Lindblad resonances. Gas and stars move through these standing waves with corotation approximately at mid-radius in the spiral.

Young stars concentrate in spiral arms because the gas concentrates there. Spiral arms are dense and promote more gravitational instabilities and cloud collisions than the interarm regions, triggering molecular cloud formation and conglomeration in the arms. The star formation rate per unit area is high in the arms as a result. This excess star formation rate is mostly in proportion to the extra molecular gas column density there, without a significant change in the star formation rate per unit molecular gas mass. The total galactic star formation rate in the main disk is not significantly enhanced by the presence of spiral arms. That is like saying the gas would have formed the same abundance of molecular clouds even without the arms. Outer disks may be different. They may have an excess of total star formation if there are spiral arms there, but this excess has not been observed yet. The difference between inner disks and outer disks is that inner disks are highly molecular and star formation in the gas is virtually saturated. Outer disks are mostly atomic and without star formation, so triggering a higher rate of star 
formation might be possible with dynamical disturbances.

\section{References}

Beck, R. 2007, A\&A, 470, 539

Bertin, G., Lin, C. C., Lowe, S. A., \& Thurstans, R. P. 1989, ApJ, 338, 104

Bigiel, F., Leroy, A., Walter, F., Brinks, E., de Blok, W. J. G., Madore, B., \& Thornley, M. D. 2008, AJ, 136, 2846

Boulanger, F., \& Viallefond, F. 1992, A\&A, 266, 37

Bournaud, F., Duc, P.-A., Brinks, E., Boquien, M., Amram, P., Lisenfeld, U., Koribalski, B.S., Walter, F., \& Charmandaris, V. 2007, Sci., 316, 1166

Bournaud, F., Elmegreen, B.G., Teyssier, R., Block, D.L., \& Puerari, I. 2010, MNRAS, in press, arXiv:1007.2566.

Braun, R., Heald, G., \& Beck, R. 2010, A\&A, 514, 42

Buta, R., \& Combes, F. 1996, Fund.Cosmic Phys., 17, 95

Dobbs, C. L., \& Pringle, J. E. 2009, MNRAS, 396, 1579

Elmegreen, B.G. 1979, ApJ, 231, 372

Elmegreen, B.G. 1987, ApJ, 312, 626

Elmegreen, B.G. 1992, in SAAS FEE Lectures, "The Galactic Interstellar Medium," with W.B. Burton and R. Genzel, ed. D. Pfenniger and P. Bartholdi, Berlin: Springer, p. 157

Elmegreen, B.G. 1994, ApJL, 425, 73

Elmegreen, B.G. 2002, ApJ, 577, 206

Elmegreen, B.G. 2007, ApJ, 668, 1064

Elmegreen, B.G., \& Elmegreen, D.M. 1983, MNRAS, 203, 31

Elmegreen, B.G., \& Elmegreen, D.M. 1986, ApJ, 311, 554

Elmegreen, B.G., \& Elmegreen, D.M. 1987, ApJ, 320, 182

Elmegreen, B.G., Elmegreen, D.M., \& Montenegro, L. 1992, ApJS, 79, 37

Elmegreen, B.G., Wilcots, E., \& Pisano, D. J. 1998, ApJ, 494, L37

Engargiola, G., Plambeck, R. L., Rosolowsky, E., \& Blitz, L. 2003, ApJS, 149, 343

Foyle, K. Rix, H.-W., Walter, F., \& Leroy, A. 2010, arXiv:1010.0678 
Fuchs, B., Dettbarn, C., \& Tsuchiya, T. 2005, A\&A, 444, 1

Grabelsky, D. A., Cohen, R. S., Bronfman, L., Thaddeus, P., \& May, J. 1987, ApJ, 315,122

Karataeva, G. M., Drozdovsky, I. O., Hagen-Thorn, V. A., Yakovleva, V. A., Tikhonov, N. A., \& Galazutdinova, O. A. 2004, AJ, 127, 789

Kennicutt, R.C., Jr. 1998, ApJ, 498, 541

Kim, W.-T., \& Ostriker, E.C. 2001, ApJ, 559, 70

Kormendy, J., \& Norman, C. A. 1979, ApJ, 233, 539

Lada, C.J., Margulis, M., Sofue, Y., Nakai, N., \& Handa, T. 1988, ApJ, 328, 143

Lau, Y.Y., Lin, C.C. \& Mark, J.W.-K. 1976, Pub. National. Acad. Sciences, 73, 1379

La Vigne, M.A., Vogel, S.N., \& Ostriker, E.C. 2006, ApJ, 650, 818

Leroy, A.K., Walter, F., Brinks, E., Bigiel, F., de Blok, W. J. G., Madore, B., \& Thornley, M. D. 2008, AJ, 136, 2782

Lin, C. C., Shu, F.H. 1964, ApJ, 140, 646

Lindblad, B., 1962, IAUS, 15, 146

Lord, S.D., \& Kenney, J.D.P. 1991, ApJ, 381, 130

Mark, J.W.-K. 1974, ApJ, 193, 539

McGee, R. X., \& Milton, J.A. 1964, Aust.J.Phys., 17, 128

Mendes de Oliveira, C., Cypriano, E. S., Sodré, L., Jr., \& Balkowski, C. 2004, ApJ, 605, 17

Morgan, W. W., Whitford, A. E., \& Code, A. D. 1953, ApJ, 118, 318

Rand, R.J., \& Kulkarni, S.R. 1990, ApJ, 349, L43

Roberts, W. W. 1969, ApJ, 158, 123

Shetty, R., Vogel, S.N., Ostriker, E.C., \& Teuben, P.J. 2007, ApJ, 665, 1138

Tamburro, D., Rix, H.-W., Walter, F., Brinks, E., de Blok, W. J. G., Kennicutt, R. C., \& Mac Low, M.-M. 2008, AJ, 136, 2872

Thomasson, M., Donner, K.H., \& Elmegreen, B.G. 1992, A\&A, 250, 316

Toomre, A. 1964, ApJ, 139, 1217

Toomre, A. 1969, ApJ, 158, 899 
Toomre, A. 1981, in "The structure and evolution of normal galaxies," Cambridge: Cambridge University Press, p. 111.

Toomre, A., \& Toomre, J. 1972, ApJ, 178, 623

Toomre, A., \& Kalnajs, A. J. 1991, in Dynamics of Disc Galaxies: Varberg Castle Sweden, p. 341

Walter, F., Brinks, E., de Blok, W.J.G., Bigiel, F., Kennicutt, R.C., Jr., Thornley, M.D., \& Leroy, A. 2008, AJ, 136, 2563 